\title{
Sex Determination Using the Second Cervical Vertebra-A Test of the Method*
}

\begin{abstract}
Sex is one of the critical questions addressed when unidentified skeletal remains are discovered in forensic or archeological contexts. Continuous testing and re-evaluation of existing techniques is essential to improve accuracy and precision. The Wescott $(J$ Forensic Sci 2000;45(2):462-6) method of sex determination from dimensions of the second cervical vertebra was blind-tested on 153 adult individuals from the Spitalfields documented collection of human skeletal remains held at the Natural History Museum, London. Significant sex differences were determined for all dimensions measured (independent two-sample $t$-test, $p<0.05-0.001$ ). The discriminant functions developed by Wescott were shown to have an overall accuracy of classification of $76.99 \%$. Using stepwise discriminant analysis, a discriminant function based on the Spitalfields data correctly classified sex in $83.3 \%$ of individuals and was able to classify males and females with equal accuracy. Additional discriminant functions are presented for use in instances where preservation of the second cervical vertebra is poor.
\end{abstract}

KEYWORDS: forensic science, forensic anthropology, sex determination, cervical vertebrae, discriminant function, Spitalfields

Reliable determination of sex from human skeletal remains is of fundamental importance, both for personal identification in forensic cases and for studies of paleodemography using archeological populations (1). The most reliable technique for estimating the sex of an individual is by examination of morphological characteristics of the bony pelvis (2-4). However, skeletal remains found in archeological or forensic contexts are often damaged or incomplete due to conditions within the burial environment or other events, such as disarticulation, scattering, and commingling. Because of these factors, it is essential that methods are developed that allow the estimation of sex from a wide range of skeletal elements.

The vertebrae have so far produced variable results when investigated for the presence of sexually dimorphic indicators $(5,6)$. Studies have primarily involved metric analysis, relating to the observation that a later growth spurt in vertebral height and greater growth in transverse diameter in males both give rise to sexual dimorphism in vertebral shape and proportions (7). Wescott (8) developed a metric method of adult sex determination based on eight measurements of the second cervical vertebra. Specimens were taken from the Hamann-Todd and Terry modern anatomical collections and consisted of 100 black and 100 white individuals of each sex ranging in age from 20 to 79 years. Five discriminant functions were formulated using a stepwise discriminant analysis procedure that selected the variables that had the greatest amount of discriminating ability (8). When tested on the sample employing a cross-validation procedure, sex was determined accurately for

\footnotetext{
${ }^{1}$ Department of Archaeological, Geographical and Environmental Sciences, School of Life Sciences, University of Bradford, Richmond Road, Bradford, West Yorkshire, BD7 1DP.

${ }^{2}$ Department of Anthropology, 308 Condon Hall, 1218 University of Oregon, Eugene, OR 97403-1218.

*Presented as a poster at the 76th American Association of Physical Anthropology Conference, March 28-31, 2007, in Philadelphia, PA.

Received 31 Aug. 2009; and in revised form 22 Oct. 2009; accepted 24 Oct. 2009.
}

$89 \%$ of the white specimens, $81 \%$ of the black specimens, and within the range $81.7-83.4 \%$, depending on the function, when all specimens were considered collectively (8).

The purpose of this study is to re-evaluate Wescott's method for estimating sex from the second cervical vertebra to (i) test its ability to accurately assess the biological sex of individuals from a documented archeological period and (ii) develop additional discriminant functions for sexing the second cervical vertebra that are specific to skeletal remains from an archeological period.

\section{Materials and Methods}

The study is based on the adult individuals from the Spitalfields collection of documented skeletal remains housed at the Natural History Museum, London, U.K. The collection spans the 18th and 19th centuries and comprises an immigrant population of European ancestry (French Huguenots) (9). Wescott's (8) eight proposed sexually dimorphic dimensions of the second cervical vertebra plus an additional dimension (width of vertebral foramen; WVF) (Table 1; Fig. 1) were measured for 153 known-sex individuals, ranging in age from 21 to 92 years. Measurements were taken to the nearest $0.1 \mathrm{~mm}$ using digital sliding calipers. For bilateral structures, the left side was recorded unless significantly damaged. Pathological specimens were not included. Wescott's (8) five original discriminant functions (Table 2) were blind-tested using the collected measurements. In instances where sex determination was not unanimous, a conclusion was reached based on majority and the level of accuracy assigned to each function.

Being able to reliably replicate measurements is an essential component of any metric study; therefore, a test of intra-observer error was conducted using a randomly selected subsample of $20 \%$ of the study population $(n=30)$. All measurements were re-collected, and the difference between them was utilized to calculate a percentage of intra-observer error. The paired-samples $t$-test was used to determine significance. 
TABLE 1-Sexually dimorphic dimensions of the second cervical vertebra as proposed by Wescott ([8]; with additions).

\begin{tabular}{|c|c|}
\hline Dimension & Definition \\
\hline Maximum sagittal length (XSL) & $\begin{array}{l}\text { The sagittal length of the vertebra from the most anterior point on the body to the most posterior point on the } \\
\text { spinous process }\end{array}$ \\
\hline Maximum height of dens (XDH) & The height from the most inferior edge of the anterior border of the body to the most superior point on the dens \\
\hline Dens sagittal diameter (DSD) & The maximum sagittal (antero-posterior) diameter of the dens \\
\hline Dens transverse diameter (DTD) & The diameter of the dens measured perpendicular to the sagittal diameter \\
\hline Length of vertebral foramen (LVF) & The internal length of the vertebral foramen, measured at the inferior edge of the foramen in the median plane \\
\hline Width of vertebral foramen (WVF) & $\begin{array}{l}\text { The maximum internal width of the vertebral foramen, wherever it may occur, measured perpendicular to the } \\
\text { median plane }\end{array}$ \\
\hline $\begin{array}{l}\text { Maximum breadth across } \\
\text { superior facets (SFB) }\end{array}$ & The maximum breadth between the most lateral edges of the superior articular facets \\
\hline Superior facet sagittal diameter (SFS) & The maximum sagittal diameter of the superior articular facet \\
\hline Superior facet transverse diameter (SFT) & The maximum transverse diameter of the superior articular facet measured perpendicular to the sagittal diameter \\
\hline
\end{tabular}

A
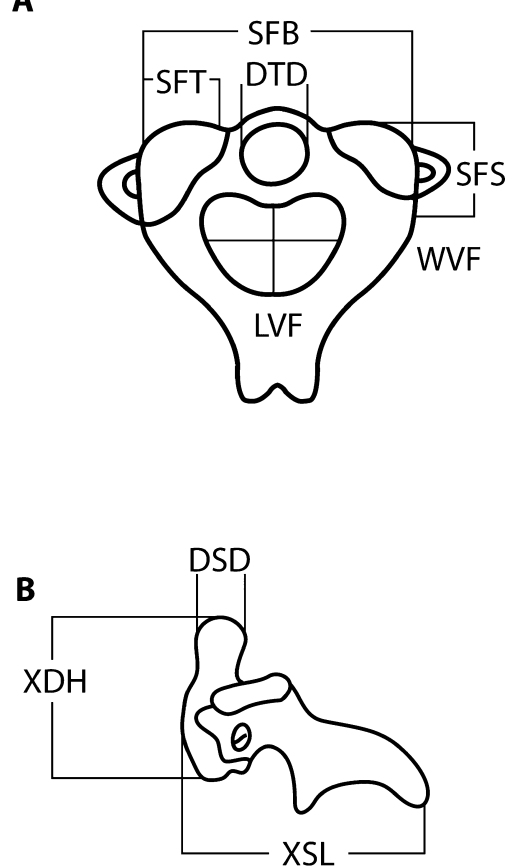

FIG. 1-Line drawing of the second cervical vertebra from: (A) a superior view, (B) a lateral view, illustrating measurements used in the earlier and present study (Fig. 1 from Wescott [8] with additions).

TABLE 2-Discriminant function equations for predicting sex from the second cervical vertebra, formulated by Wescott (8).

\begin{tabular}{ll}
\hline Function & \multicolumn{1}{c}{ Equation* } \\
\hline 1 & $(\mathrm{XSL} \times 0.6488)-32.159$ \\
2 & $(\mathrm{XSL} \times 0.5836)+(\mathrm{SFS} \times 0.4359)-36.6899$ \\
3 & $(\mathrm{XSL} \times 0.5490)+(\mathrm{SFS} \times 0.3234)+(\mathrm{SFT} \times 0.3021)-38.0016$ \\
4 & $(\mathrm{XSL} \times 0.52882)+(\mathrm{SFS} \times 0.2987)+(\mathrm{SFT} \times 0.2796)$ \\
& $-(\mathrm{LVF} \times 0.1694)-36.3804$ \\
5 & $(\mathrm{XSL} \times 0.5343)+(\mathrm{SFS} \times 0.3005)+(\mathrm{SFT} \times 0.2142)$ \\
& $-(\mathrm{LVF} \times 0.1671)+(\mathrm{XDH} \times 0.1183)-37.1515$ \\
\hline
\end{tabular}

LVF, length of vertebral foramen; SFS, superior facet sagittal diameter; SFT, superior facet transverse diameter; XSL, maximum sagittal length; $\mathrm{XDH}$, maximum height of dens.

*Sectioning point: 0 . Male $>0$; Female $<0$.

\section{Statistical Approach}

Discriminant function analysis, a procedure that maximizes within-group differences, was utilized to evaluate the effectiveness of the second cervical vertebra at estimating sex. The primary goals of this procedure are to find the dimension or dimensions along which groups differ and to find classification functions to predict group membership (10). A linear combination of variables is generated that maximizes the probability of correctly assigning observations to predetermined groups (11), assuming that the two populations (male and female) are multivariate normal with equal covariance matrices (12).

SPSS 13.0 (SPSS Inc., Chicago, IL) was utilized to run direct and stepwise discriminant analysis. In direct discriminant analysis, all predictors enter the equations at once, and each predictor is assigned only the unique association it has with the groups. Variance shared among predictors contributes to the total relationship, but not to any one predictor (10). In contrast, the stepwise procedure selects a subset of variables based on the squared partial correlation and the significance level from an analysis of covariance that has the greatest amount of discriminating ability (8). In other words, the statistical criteria alone determine the order of entry (13).

To evaluate the discriminating ability of the variables selected, a cross-validation (jack-knife) procedure was employed. This procedure classifies each individual case based on discriminant analyses run on all other cases; thus, the case being classified does not influence the discriminant analysis used to classify it (8). In this way, a more realistic estimate is gained of the ability of predictors to separate groups (10).

\section{Results}

\section{Accuracy of Wescott Functions}

The assessment of accuracy involved sex determination using the five discriminant functions developed by Wescott (8). A total of 113 of the 153 individuals in the sample population were suitable for inclusion in the accuracy assessment. Of these, 87 individuals were correctly assigned their documented sex, resulting in an overall accuracy of classification of $76.99 \%$. The percent of males and females correctly classified by individual functions ranged from 70.91 to $78.90 \%$ (Table 3). Where functions could not be applied, it was generally the result of loss of the spinous process, which precluded measurement of the maximum sagittal length (XSL).

\section{Analysis of Spitalfields Data}

The result of the intra-observer error test (Table 4) indicates that overall the measurements used in this study are replicable. The average percent intra-observer error is 1.22 , with no individual measurement exceeding 2\%. Only length of vertebral foramen (LVF), dens sagittal diameter (DSD), and superior facet transverse diameter (SFT) exhibit relatively high intra-observer error, which, with the exception of SFT, is in accordance with the findings of 
TABLE 3-Classification accuracy using the Wescott discriminant functions.

\begin{tabular}{lcc}
\hline Classification Accuracy $(\%)$ & & \\
\hline Function & Spitalfields & Original \\
\hline 1 & 71.68 & 81.70 \\
2 & 75.89 & 83.40 \\
3 & 74.11 & 82.90 \\
4 & 70.91 & 83.10 \\
5 & 78.90 & 82.00 \\
\hline
\end{tabular}

TABLE 4-Intra-observer error results.

\begin{tabular}{lccc}
\hline Variable & $N$ & Percent Error* & Absolute Range $(\mathrm{mm})$ \\
\hline XSL & 24 & 0.61 & $0.09-1.40$ \\
XDH & 29 & 0.69 & $0.00-4.99$ \\
DSD & 30 & 1.94 & $0.00-6.09$ \\
DTD & 30 & 1.55 & $0.00-6.80$ \\
LVF & 28 & 1.97 & $0.00-7.50$ \\
WVF & 29 & 0.49 & $0.00-1.71$ \\
SFB & 28 & 0.13 & $0.00-1.32$ \\
SFS & 30 & 1.70 & $0.00-5.56$ \\
SFT & 30 & 1.94 & $0.00-9.20$ \\
\hline
\end{tabular}

XSL, maximum sagittal length; XDH, maximum height of dens; DSD, dens sagittal diameter; DTD, dens transverse diameter; LVF, length of vertebral foramen; WVF, width of vertebral foramen; SFB, maximum breadth across superior facets; SFS, superior facet sagittal diameter; SFT, superior facet transverse diameter.

*Average percent error: 1.22 .

TABLE 5-Descriptive statistics for Spitalfields sample.

\begin{tabular}{lccccc}
\hline & \multicolumn{2}{c}{ Male } & \multicolumn{2}{c}{ Female } & \\
\cline { 2 - 5 } Variable & Mean & SD & Mean & SD & $t$-value \\
\hline XSL & 50.215 & 2.4531 & 46.498 & 2.7227 & $7.413^{*}$ \\
XDH & 38.972 & 2.2586 & 36.183 & 2.0856 & $7.431^{*}$ \\
DSD & 11.862 & 0.8053 & 11.198 & 0.6658 & $5.457^{*}$ \\
DTD & 10.649 & 0.7829 & 10.053 & 0.8947 & $3.629^{*}$ \\
LVF & 16.506 & 1.9427 & 15.767 & 1.4651 & $2.816 \dagger$ \\
WVF & 23.336 & 1.3310 & 23.121 & 1.4804 & $2.402^{*}$ \\
SFB & 46.313 & 2.7316 & 42.622 & 2.1776 & $9.272^{*}$ \\
SFS & 17.511 & 1.5394 & 16.450 & 1.1653 & $3.543^{*}$ \\
SFT & 17.351 & 1.4949 & 15.831 & 1.2925 & $6.402^{*}$ \\
\hline
\end{tabular}

XSL, maximum sagittal length; XDH, maximum height of dens; DSD, dens sagittal diameter; DTD, dens transverse diameter; LVF, length of vertebral foramen; WVF, width of vertebral foramen; SFB, maximum breadth across superior facets; SFS, superior facet sagittal diameter; SFT, superior facet transverse diameter.

*Highly significant.

${ }^{\dagger}$ Very significant.

'Significant.

Wescott (8). However, the results of the paired-samples $t$-test indicate that the differences between the original and re-taken measurements of LVF and superior facet sagittal diameter (SFS) were significant at the 5\% level ( $p=0.027$ and $p=0.031$, respectively).

A total of 105 individuals (47 male and 58 female) for which a complete set of nine measurements could be taken were included in the analysis. Descriptive statistics for males and females are presented in Table 5. Significant sex differences were present for all of the nine variables, as determined by the independent two-sample $t$-test (Table 5).
TABLE 6-Summary of stepwise selection.

\begin{tabular}{lcccc}
\hline & & \multicolumn{3}{c}{ Wilks' Lambda } \\
\cline { 3 - 5 } Step & Variable Entered & Statistic & Exact $F$ & Significance \\
\hline 1 & SFB & 0.634 & 59.357 & 0.000 \\
2 & XSL & 0.563 & 39.648 & 0.000 \\
3 & WVF & 0.538 & 28.860 & 0.000 \\
4 & DSD & 0.517 & 23.369 & 0.000 \\
\hline
\end{tabular}

SFB, maximum breadth across superior facets; XSL, maximum sagittal length; WVF, width of vertebral foramen; DSD, dens sagittal diameter.

TABLE 7-Unstandardized canonical discriminant function coefficients.

\begin{tabular}{lc}
\hline Variable & Function 1 Coefficients* \\
\hline XSL & 0.210 \\
DSD & 0.406 \\
WVF & -0.233 \\
SFB & 0.255 \\
Constant & -20.651 \\
\hline
\end{tabular}

XSL, maximum sagittal length; DSD, dens sagittal diameter; WVF, width of vertebral foramen; SFB, maximum breadth across superior facets.

$*$ Sectioning point: 0.0. Males: $>0.0$; females: $<0.0$.

TABLE 8-Classification results.

\begin{tabular}{ccccc}
\hline & & \multicolumn{3}{c}{ Predicted Group Membership } \\
\cline { 3 - 5 } & Sex & Male & Female & Total \\
\hline \multirow{2}{*}{ Count $\%$} & Male & 40 & 8 & 48 \\
& Female & 10 & 50 & 60 \\
& Male & 83.3 & 16.7 & 100.0 \\
& Female & 16.7 & 83.3 & 100.0 \\
\hline
\end{tabular}

Stepwise selection was employed to develop a model of measurement that displays the maximum amount of discriminating ability (Table 6). At each step, up to a maximum of 18 , the variable that minimizes the overall Wilks' Lambda is entered. The minimum partial $F$ to enter and maximum partial $F$ to remove were kept at the default values of 3.84 and 2.71 , respectively. The $F$ to remove value tests the significance of the decrease in discrimination should that variable be removed. By exceeding the $F$-ratio value, maximum breadth across superior facets (SFB), XSL, WVF, and DSD were not removed from the analysis and therefore form the basis of the discriminant function (Table 7). Of these, SFB is the single best variable for estimating sex.

An eigenvalue of 0.935 indicates that the function is a strong one and accounts for $100 \%$ of the variance. Testing of the function produced Wilks' Lambda and chi-square values of 0.517 and 66.658 , respectively. These results indicate that the function is highly significant $(p=0.000)$. Within-group means for each canonical variable (female and male) were -0.862 and 1.018 , respectively. Overall, $83.3 \%$ of original grouped cases were correctly classified. Males and females were classified with equal success (Table 8). When tested on the sample employing a cross-validation procedure, the percent of males and females correctly classified was $79.2 \%$ and $83.3 \%$, respectively.

One particular issue that deserves further attention is the applicability of the method in instances where the second cervical vertebra has been damaged in the postdepositional environment. An important observation made when collecting data on the Spitalfields population was that when damage occurs, it is the spinous process 
TABLE 9-Discriminant analysis equations for predicting sex from fragmentary second cervical vertebrae.

\begin{tabular}{|c|c|c|c|c|c|}
\hline Variable & Function 1 & Function 2 & Function 3 & Function 4 & Function 5 \\
\hline DSD & 1.248 & 0.593 & 0.569 & 0.545 & 0.485 \\
\hline WVF & 0.184 & 0.026 & 0.025 & 0.024 & -0.116 \\
\hline $\mathrm{XDH}$ & & 0.340 & 0.305 & 0.290 & 0.211 \\
\hline SFS & & & 0.147 & 0.150 & 0.113 \\
\hline DTD & & & & 0.096 & 0.067 \\
\hline LVF & & & & & 0.152 \\
\hline SFT & & & & & 0.302 \\
\hline Constant & -18.608 & -20.184 & -21.061 & -21.249 & -20.806 \\
\hline $\begin{array}{l}\text { Sectioning } \\
\text { point* }\end{array}$ & 0.0 & 0.0 & 0.0 & 0.0 & 0.0 \\
\hline $\begin{array}{l}\text { Males classified } \\
\text { correctly, } \%\end{array}$ & 68.1 & 73.9 & 74.6 & 76.1 & 71.9 \\
\hline $\begin{array}{l}\text { Females } \\
\text { classified } \\
\text { correctly, \% }\end{array}$ & 67.9 & 76.3 & 77.5 & 78.8 & 83.3 \\
\hline $\begin{array}{l}\text { Total classified } \\
\text { correctly, } \%\end{array}$ & 68.0 & 75.2 & 76.2 & 77.6 & 78.2 \\
\hline
\end{tabular}

DSD, dens sagittal diameter; WVF, width of vertebral foramen; XDH, maximum height of dens; SFS, superior facet sagittal diameter; DTD, dens transverse diameter; LVF, length of vertebral foramen; SFT, superior facet transverse diameter.

*Sectioning point: 0.0. Males: $>0.0$; females: $<0.0$.

and superior articular facets that are most commonly affected. This precluded measurement of the XSL and maximum breadth across the SFB, which was a problem for two reasons: (i) all of the discriminant functions developed by Wescott (8) require measurement of the XSL and (ii) discriminant function analysis of the Spitalfields data revealed XSL and SFB to be the variables with the greatest amount of discriminating ability. As a result, fragmentary specimens could not be sexed using this method, which limits its usefulness both in forensic cases and for archeological samples.

To overcome this issue, several direct discriminant analyses were run that excluded XSL and SFB and included variables that were less commonly damaged. The resulting discriminant functions are presented in Table 9.

\section{Discussion}

The results of this blind test confirm the earlier findings of Wescott (8) that metric analysis of the second cervical vertebra can accurately determine the sex of human skeletal remains. The discriminant function generated from the Spitalfields data using a stepwise procedure was able to estimate sex with an accuracy of $83.3 \%$ and was able to classify males and females with equal accuracy (Table 8). Methods of sex assessment are generally considered useful if they produce accuracies of at least $80 \%$ (14). Although the second cervical vertebra would never be used as the sex indicator of choice in a near-complete skeletal assemblage, the results of this study indicate that it can be used to correctly classify sex with nearly the same level of accuracy as other traditionally used single bones. The forearm bones, for example, can predict sex with an accuracy of between $76 \%$ and $86 \%$ (15), the tibia with $92.2 \%$ (16), the humerus with between $76.8 \%$ and $95.5 \%$ (17), the first cervical vertebra within the range $75-81 \%$ depending on the function used (5), and for the twelfth thoracic and first lumbar vertebrae, various discriminant functions yielded accuracies ranging from c. $77 \%$ to $100 \%$ for the Spitalfields collection and $85-92 \%$ for the Terry Collection (6).

A decreased overall accuracy of classification was obtained in this study than was recorded in Wescott's study, which is believed to be a result of population variability. It is well known that human proportions vary systematically between populations. These differences can significantly affect metric determinations of sex because of the dependence of measurement methods on absolute size differences of males and females (18).

The most reliable estimates of sex are therefore obtained when the population being investigated is as similar as possible in proportions to the reference population used to create the method. In this study, four dimensions of the second cervical vertebra were selected as having the greatest power of discrimination between the sexes (Table 6). With the exception of XSL, none of these dimensions were found to have significant discriminating ability in Wescott's study. This demonstrates the different patterns of sexual dimorphism exhibited in different populations and highlights the importance of considering geographical and temporal separation when choosing methods of sex determination in skeletal remains.

The results in Table 6 indicate that SFB is the single best discriminator of sex in the Spitalfields sample, compared with XSL in Wescott's study. Other work has demonstrated the presence of sexual dimorphism in the occipital condyles and foramen magnum region of the skull $(19,20)$, and articular facets and vertebral foramen of the first cervical vertebra (5). Such observations can be attributed to the functional relationship between the cranial base and first two cervical vertebrae as load-bearing regions, and sex differences in the size and weight of the brain and skull resulting in sexual dimorphism of these structures. Autopsy data indicate that while male brain weight increased by an average of $0.66 \mathrm{~g}$ per year from a mean of $1372 \mathrm{~g}$ for those born in 1860 , to $1424 \mathrm{~g}$ for those born in 1940, female brain weight did not increase appreciably until 1900 (21). Secular trends in brain weight may therefore explain why different dimensions showed greater or lesser degrees of sexual dimorphism in the Spitalfields population compared with a more recent dissecting-room population sampled by Wescott.

A study examining the relative survival of skeletal elements indicated that the second cervical vertebra, in conjunction with the proximal femur, is more likely to survive postdepositional processes than any other single bone traditionally used for metric sexing (22). When preservation of the second cervical vertebra is poor, it is the spinous process and superior articular facets that are most commonly affected. To extend the applicability of the present method to cases of fragmentary vertebrae, direct discriminant analysis was performed that excluded XSL and SFB and created functions using dimensions less commonly damaged (Table 9). Although the resulting functions predict sex with a lesser degree of accuracy (68-78\%), they may be of particular importance in instances where a skeletal assemblage is highly fragmentary and other methods of sex determination are not available for use.

The results of the intra-observer error test suggest that the measurements used in this study are replicable. Only LVF, DSD, and SFT exhibit moderately high percent error; however, no individual value exceeds $2 \%$. Of these, LVF and DSD were also demonstrated to have relatively high values of both intra-observer error and interobserver error in Wescott's study. Only the dimension DSD was selected by the stepwise procedure as contributing to the discriminating ability of the second cervical vertebra. The variable with the greatest power of discrimination, SFB, has a very low level of intra-observer error $(0.13 \%$; Table 4$)$. Despite the low levels of percent error, the differences between the original and re-taken measurements of LVF and SFS were found to be statistically significant. This may have been attributable to the effect of extreme outliers; these dimensions were found to be among those exhibiting the greatest absolute ranges (data not shown). Neither dimension was selected by stepwise discriminant analysis and therefore did 
not contribute to the discriminating ability of the function shown in Table 7.

In conclusion, the results of this blind test indicate that the method of sex determination from dimensions of the second cervical vertebra proposed by Wescott (8) possesses a significant ability to discriminate between the sexes. The results of this study further confirm that the method is applicable to both modern human skeletal remains and those from archeological contexts. A discriminant function equation is presented that is able to classify males and females with a level of accuracy that rivals that of some other traditionally used single bones and would be of particular value for determining sex in individuals of European ancestry. Additional functions are presented for use in instances where preservation of the skeleton is poor and other methods of sex determination are not available for use.

\section{Acknowledgments}

The authors are indebted to Mr. Rob Kruszynski of the Natural History Museum for granting access to the Spitalfields documented collection of human remains. Appreciation is also extended to $\mathrm{C}$. Knüssel and S. Black for useful comments on the M.Sc. dissertation of EJM from which the present research was extrapolated.

\section{References}

1. Mays S, Cox M. Sex determination in skeletal remains. In: Cox M Mays S, editors. Human osteology in archaeology and forensic science. London, UK: Greenwich Medical Media Ltd, 2000;117-30.

2. Phenice TW. A newly developed visual method of sexing the os pubis. Am J Phys Anthropol 1969;30:297-302.

3. Ubelaker DH, Volk CG. A test of the phenice method for the estimation of sex. J Forensic Sci 2002;47(1):19-24.

4. Durić M, Rakočević Z, Donić D. The reliability of sex determination of skeletons from forensic context in the Balkans. Forensic Sci Int 2005;147:159-64.

5. Marino EA. Sex estimation using the first cervical vertebra. Am J Phys Anthropol 1995;97:127-33.

6. Pastor RF. Sexual dimorphism in vertebral dimensions at the T12/L1 junction. Proceedings of the American Academy of Forensic Sciences; 2005 Feb 21-26; New Orleans, LA. Denver, CO: Publication Printers, $2005 ; 11: 302-3$.
7. Taylor JR, Twomey LT. Sexual dimorphism in human vertebral body shape. J Anat 1984;138:281-6.

8. Wescott DJ. Sex variation in the second cervical vertebra. J Forensic Sci 2000;45(2):462-6.

9. Cox M. Life and death in Spitalfields 1700 to 1850 . York, UK: British Library Cataloguing in Publication Data, 1996.

10. Tabachnick BG, Fidell LS. Using multivariate statistics, 2nd edn. New York, NY: Harper \& Row Publishers, 1989.

11. Quinn GP, Keough MJ. Discriminant function analysis. In: Quinn GP, Keough MJ, editors. Experimental design and data analysis for biologists. Cambridge, UK: Cambridge University Press, 2002;435-42.

12. Press SJ, Wilson S. Choosing between logistic regression and discriminant analysis. J Am Stat Assoc 1978;73:699-705.

13. Kinnear PR, Gray CD. SPSS for Windows Made Simple Release 10. Sussex, UK: Psychology Press Ltd, 2000.

14. Rogers TL. A visual method of determining the sex of skeletal remains using the distal humerus. J Forensic Sci 1999;44(1):57-60.

15. Barrier IL, L'Abbé EN. Sex determination from the radius and ulna in a modern South African sample. Forensic Sci Int 2008;179(1):85. e1-7

16. Šlaus M, Tomičić Ž. Discriminant function sexing of fragmentary and complete tibiae from medieval Croatian sites. Forensic Sci Int 2005; 147:147-52.

17. Frutos LR. Metric determination of sex from the humerus in a Guatemalan forensic sample. Forensic Sci Int 2005;147:153-7.

18. Albanese J. A metric method for sex determination using the hipbone and the femur. J Forensic Sci 2003;48(2):263-73.

19. Gapert R, Black S, Last J. Sex determination from the occipital condyle: discriminant function analysis in an eighteenth and nineteenth century British sample. Am J Phys Anthropol 2009;138:384-94.

20. Holland TD. Sex determination of fragmentary crania by analysis of the cranial base. Am J Phys Anthropol 1986;70:203-8.

21. Miller AKH, Corsellis JAN. Evidence for secular change in human brain weight during the past century. Ann Hum Biol 1977;4:253-7.

22. Waldron $\mathrm{T}$. The relative survival of the human skeleton: implications for palaeopathology. In: Boddington A, Garland A-C, Janaway RC, editors. Death, decay and reconstruction. Manchester, UK: Manchester University Press, 1987;55-64.

Additional information and reprint requests:

Emily J. Marlow, M.Sc.

The University of Manchester

Faculty of Life Sciences

Stopford Building

Oxford Road

Manchester

M13 9PT

U.K.

E-mail: marlow_emily@hotmail.com 